\title{
INDUCTANCE AND FORCE CALCULATION FOR AX- ISYMMETRIC COIL SYSTEMS INCLUDING AN IRON CORE OF FINITE LENGTH
}

\section{T. Lubin*, K. Berger, and A. Rezzoug}

Groupe de Recherche en Electrotechnique et Electronique de Nancy, Université de Lorraine, Green, EA 4366, Vandœuvre-lès-Nancy F54506, France

\begin{abstract}
This paper presents new semi-analytical expressions to calculate the self-inductance and the electromagnetic force for a ferromagnetic cylinder of finite length placed inside a circular coil of rectangular cross section. The proposed analytical model is based on boundary value problems with Fourier analysis. Laplace's and Poisson's equations are solved in each region by using the separation of variables method. The boundary and continuity conditions between the different regions yield to the global solution. Moreover, the iron cylinder is assumed to be infinitely permeable. Magnetic field distribution, self-inductance and electromagnetic force obtained with the proposed analytical model are compared with those obtained from finite-element.
\end{abstract}

\section{INTRODUCTION}

Circular coils are widely used in many industrial applications such as tubular actuators, transformers, linear accelerators, magnetic valves, induction heaters, magnetic resonance imaging. An accurate knowledge of the magnetic field distribution is necessary for the computation of useful quantities such as self and mutual inductances, stored energy and electromagnetic forces. The magnetic field can be evaluated by analytical methods or by numerical techniques like finite elements. Finite elements simulations give accurate results considering the nonlinearity of ferromagnetic materials for iron-cored coils. However, this method is computer time consuming and poorly flexible for the first step of design stage. Analytical models can provide

Received 11 May 2012, Accepted 13 June 2012, Scheduled 22 June 2012

* Corresponding author: Thierry Lubin (thierry.lubin@univ-lorraine.fr). 
closed-form solutions giving physical insight for designers. They are useful tools for design optimization since continuous derivatives issued from the analytical solution are of great importance in most optimization methods.

Analytical models have been proposed since a long time for computing the magnetic field distribution of ironless circular coils [115]. As the coils are in free space (without any ferromagnetic material), analysis is generally based on the Biot-Savart law. The analytical expressions of the magnetic field can be expressed in terms of elliptic integrals of the first and second kind or by integrals with the product of Bessel functions $[9,11]$. Although these methods give very accurate results, they are not suitable to study circular coils with iron-core structures.

An alternative analytical method to compute the magnetic field of circular coils with iron parts is based on boundary value problems with Fourier analysis. This method consists in solving directly the Maxwell's equations in the different regions, e.g., air-gap and coils by the separation of variables method $[16,17]$. The magnetic field solutions in each region are obtained by using boundary and interface conditions. The solutions are expressed in terms of infinite series. Boundary value problem has been used by Rabins [18] to compute the inductances of a transformer with a simplified geometry consisting of a core, coils, and yokes of infinite extent. In [19-21], the magnetic field distribution of circular coils located between two semi-infinite blocks of iron is given. In these models, the ferromagnetic parts are supposed to be infinitely permeable and are taken into account by means of the boundary conditions. In $[22,23]$, the self and mutual inductances are computed for filamentary turns placed on infinitely-long ferromagnetic core of circular cross-section. In [24,25], eddy-current problems are solved inside infinitely long conducting rods with coaxial circular coils driven by alternating current. The coils are considered as filamentary current sources. The solution is given in the form of integrals of firstorder Bessel functions. In [26,27], an analytical approach to compute eddy-currents induced in a conducting/ferromagnetic rod of finite length by a coaxial coil is developed. The authors use the truncated region eigenfunction expansion to compute the magnetic field inside the rod.

In this paper, we propose new semi-analytical expressions to compute the self inductance and the electromagnetic force in a system composed of a coil, with rectangular cross-section, and a ferromagnetic cylinder (Fig. 1). A similar approach to that presented in $[16,24,26]$ is followed to compute the magnetic field. However, compared to $[24,26]$, only the magnetostatic case is studied here (i.e., no-eddy current in the 


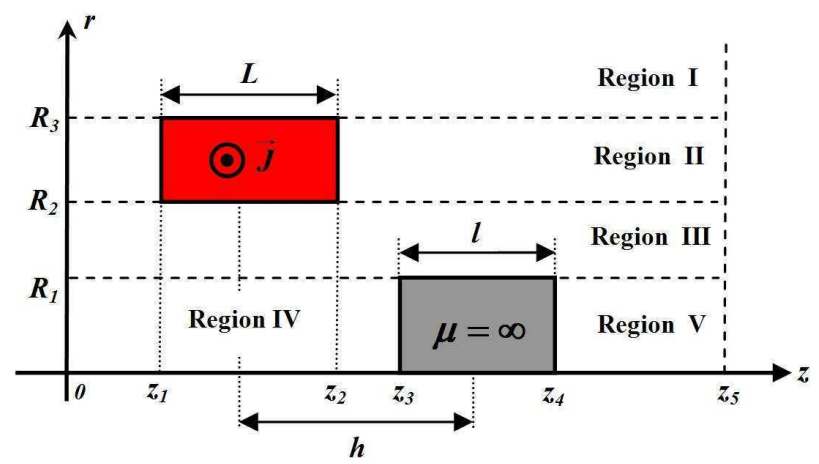

Figure 1. Axisymmetric system: a circular coil of rectangular cross section with an iron cylinder of finite length placed on the same axis at a distance $h$.

ferromagnetic cylinder). The analytical model is based on the solution of Laplace's and Poisson's equations in the different five regions as indicated in Fig. 1. The electromagnetic force acting on the iron core is obtained by using the Maxwell stress tensor method. The self-inductance is also computed with the analytical model. In order to validate the proposed model, the results are compared with those obtained from finite elements simulations.

\section{PROBLEM FORMULATION AND ASSUMPTIONS}

The geometric representation of the studied problem is shown in Fig. 1. It consists of a circular coil of rectangular cross section with inner radius $R_{2}$, outer radius $R_{3}$, and length $L=\left(z_{2}-z_{1}\right)$. This coil is fed with a uniform current density $J$ in the $\theta$-direction. An iron cylinder of radius $R_{1}$ and length $l=\left(z_{4}-z_{3}\right)$ is placed on the same axis as that of the coil. As shown in Fig. 1, the relative axial position between the center of the iron cylinder and the center of the coil is noted $h$.

The whole domain is limited in the axial direction $(z=0$ and $z=z_{5}$ ), where homogeneous Dirichlet boundary conditions have been imposed on the magnetic vector potential $(A=0)$. It is also possible to impose homogeneous Neumann boundary conditions but the global solution will be more complex. These outer boundaries must be chosen sufficiently far away from the area where reliable solutions are needed so that they do not affect the results $\left(z_{1} \gg 0\right.$ and $\left.z_{5} \gg z_{4}\right)$.

The approximation in the modeling of this problem is in assuming infinite permeability for the iron cylinder, so the tangential component of the magnetic field is null on its boundaries. 
As can be seen in Fig. 1, the whole domain of the field problem is divided into five regions: the region of air above the winding (Region I), the winding region (Region II), the air-gap between the winding and the iron core (Region III), the air region on the left of the iron core (Region IV), and the air region on the right of the iron core (Region V). As indicated previously, the iron cylinder is considered as infinitely permeable. This implies that the magnetic field is not calculated inside the iron cylinder but that the material is represented by a boundary condition at its surface. Therefore, the iron cylinder splits the surrounding space in two regions (Region IV and Region V in Fig. 1). This is not the case for the current source coil (Region II in Fig. 1) which presents the same permeability as the air.

A magnetic vector potential formulation in cylindrical coordinates is used to solve the problem. The problem being axisymmetric, the magnetic vector potential presents only one component along the $\theta$ direction and only depends on the $r$ and $z$ coordinates. From Maxwell's equations and considering the Coulomb gauge, the field equations in terms of magnetic vector potential $A$ are a Poisson equation in the coil region and a Laplace equation in the other regions

$$
\begin{cases}\nabla^{2} A_{I I}=-\mu_{0} J & \text { for Region II (coil) } \\ \nabla^{2} A_{i}=0 & \text { for Region } i=\mathrm{I}, \mathrm{III}, \mathrm{IV} \text { and } \mathrm{V}\end{cases}
$$

where $\mu_{0}$ is the permeability of the vacuum, and $J$ is the current density in the coil.

\section{ANALYTICAL SOLUTION OF THE MAGNETIC FIELD}

The solution of any partial differential equation (PDE) depends on the domain in which the solution is to be valid as well as the boundary conditions that this solution must satisfy. By using the separation of variables method, we now consider the general solution of (1) in Regions I to $\mathrm{V}$.

\subsection{General Solution of Laplace's Equation in Region I}

In Region I, we have to solve the Laplace equation in a cylinder of inner radius $R_{3}$ and infinite outer radius, delimited in the axial direction by $z=0$ and $z=z_{5}$

$$
\frac{\partial^{2} A_{I}}{\partial r^{2}}+\frac{1}{r} \frac{\partial A_{I}}{\partial r}-\frac{A_{I}}{r^{2}}+\frac{\partial^{2} A_{I}}{\partial z^{2}}=0 \quad \text { for } \quad\left\{\begin{array}{l}
R_{3} \leq r \leq \infty \\
0 \leq z \leq z_{5}
\end{array}\right.
$$


As indicated previously, homogeneous Dirichlet boundary conditions have been imposed at $z=0$ and $z=z_{5}$

$$
A_{I}(r, z=0)=0 \quad \text { and } \quad A_{I}\left(r, z=z_{5}\right)=0
$$

Moreover, the vector potential tends to zero when $r \rightarrow \infty$

$$
A_{I}(r \rightarrow \infty, z) \rightarrow 0
$$

Considering the boundary conditions (3) and (4), the general solution of (2) can be expressed as

$$
A_{I}(r, z)=\sum_{n=1}^{\infty} b_{n}^{I} K_{1}\left(\alpha_{n} r\right) \sin \left(\alpha_{n} z\right)
$$

where $n$ is a positive integer, $\alpha_{n}=n \pi / z_{5}$ are the eigenvalues, and $K_{1}$ is the modified Bessel function of the second kind and order 1 [28].

The integration constant $b_{n}^{I}$ will be determinate in Section 4 from the interface conditions between Region I and Region II.

\subsection{General Solution of Poisson's Equation in Region II}

In Region II, we have to solve the Poisson equation in a cylinder of inner radius $R_{2}$ and outer radius $R_{3}$, delimited by $z=0$ and $z=z_{5}$

$$
\frac{\partial^{2} A_{I I}}{\partial r^{2}}+\frac{1}{r} \frac{\partial A_{I I}}{\partial r}-\frac{A_{I I}}{r^{2}}+\frac{\partial^{2} A_{I I}}{\partial z^{2}}=-\mu_{0} J(r, z) \text { for }\left\{\begin{array}{l}
R_{2} \leq r \leq R_{3} \\
0 \leq z \leq z_{5}
\end{array}\right.
$$

where $J(r, z)$ is the current density distribution in Region II.

As the current density in the coil is homogeneous, the current density distribution is independent of the $r$-coordinate and depends only on the $z$-coordinate as shown in Fig. 2

$$
J(z)= \begin{cases}J & \forall z \in\left[z_{1}, z_{2}\right] \\ 0 & \text { elsewhere }\end{cases}
$$

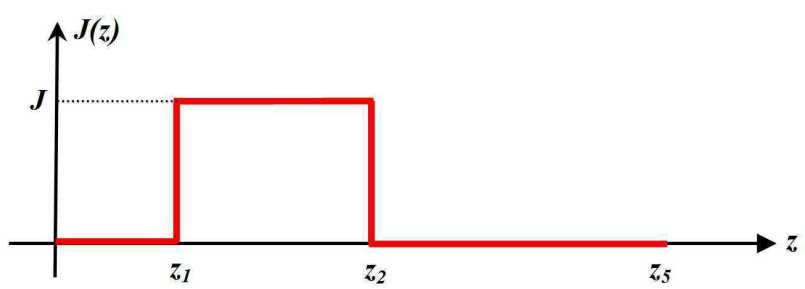

Figure 2. Current density distribution along the axial coordinate $z$ in Region II. 
As for Region I,

$$
A_{I I}(r, z=0)=0 \quad \text { and } \quad A_{I I}\left(r, z=z_{5}\right)=0
$$

Equation (6) is classically solved [29] by finding the eigenvalues and the eigenfunctions of the homogeneous equation $\left(\nabla^{2} A_{I I}=0\right)$ which satisfies the boundary conditions (8). The source term $J(z)$ can then be expanded in terms of the eigenfunctions, i.e., orthogonal basis, as

$$
\begin{aligned}
J(z) & =\sum_{n=1}^{\infty} J_{n} \sin \left(\alpha_{n} z\right) \quad \text { with } \quad J_{n}=\frac{2}{z_{5}} \int_{0}^{z_{5}} J(z) \sin \left(\alpha_{n} z\right) d z \\
J_{n} & =\frac{2 J}{n \pi}\left[\cos \left(\alpha_{n} z_{1}\right)-\cos \left(\alpha_{n} z_{2}\right)\right]
\end{aligned}
$$

The general solution of (6), which requires the solution of a nonhomogeneous Bessel's differential equation on the $r$-variable, is then given by

$$
A_{I I}(r, z)=\sum_{n=1}^{\infty}\left\{a_{n}^{I I} I_{1}\left(\alpha_{n} r\right)+b_{n}^{I I} K_{1}\left(\alpha_{n} r\right)-C_{n} L_{1}\left(\alpha_{n} r\right)\right\} \sin \left(\alpha_{n} z\right)
$$

with

$$
C_{n}=\mu_{0}(\pi / 2) J_{n} \alpha_{n}^{-2}
$$

where $n$ is a positive integer, and $I_{1}$ and $K_{1}$ are respectively the modified Bessel functions of the first and second kind of order 1, and $L_{1}$ is the modified Struve function of order 1 [28].

The integration constants $a_{n}^{I I}$ and $b_{n}^{I I}$ in (10) will be determinate in Section 4 from the interface conditions at $r=R_{2}$ and $r=R_{3}$.

\subsection{General Solution of Laplace's Equation in Region III}

In Region III, we have to solve the Laplace equation in a cylinder of inner radius $R_{1}$ and outer radius $R_{2}$, delimited by $z=0$ and $z=z_{5}$

$$
\frac{\partial^{2} A_{I I I}}{\partial r^{2}}+\frac{1}{r} \frac{\partial A_{I I I}}{\partial r}-\frac{A_{I I I}}{r^{2}}+\frac{\partial^{2} A_{I I I}}{\partial z^{2}}=0 \quad \text { for } \quad\left\{\begin{array}{l}
R_{1} \leq r \leq R_{2} \\
0 \leq z \leq z_{5}
\end{array}\right.
$$

The boundary conditions for the Region III are

$$
A_{I I I}(r, z=0)=0 \quad \text { and } \quad A_{I I I}\left(r, z=z_{5}\right)=0
$$

Considering (13), the general solution of (12) can be expressed as

$$
A_{I I I}(r, z)=\sum_{n=1}^{\infty}\left\{a_{n}^{I I I} I_{1}\left(\alpha_{n} r\right)+b_{n}^{I I I} K_{1}\left(\alpha_{n} r\right)\right\} \sin \left(\alpha_{n} z\right)
$$

where $n$ is a positive integer. The integration coefficients $a_{n}^{I I I}$ and $b_{n}^{I I I}$ in (14) will be determinate in subsection 4 from the interface conditions at $r=R_{1}$ and $r=R_{2}$. 


\subsection{General Solution of Laplace's Equation in Region IV}

As shown in Fig. 1, Region IV is delimited by a cylinder of radius $R_{1}$, and by $z=0$ and $z=z_{3}$ in the axial direction. The magnetic vector potential in Region IV satisfies to the Laplace equation

$$
\frac{\partial^{2} A_{I V}}{\partial r^{2}}+\frac{1}{r} \frac{\partial A_{I V}}{\partial r}-\frac{A_{I V}}{r^{2}}+\frac{\partial^{2} A_{I V}}{\partial z^{2}}=0 \quad \text { for } \quad\left\{\begin{array}{l}
0 \leq r \leq R_{1} \\
0 \leq z \leq z_{3}
\end{array}\right.
$$

The boundary condition at $z=0$ is

$$
A_{I V}(r, z=0)=0
$$

The radial component of the magnetic field on the side of the iron cylinder being null, the boundary condition at $z=z_{3}$ is then given by

$$
\left.\frac{\partial A_{I V}}{\partial z}\right|_{z=z_{3}}=0
$$

Moreover, the magnetic vector potential $A_{I V}$ must be finite at $r=0$. Considering the boundary conditions (16) and (17), the general solution of (15) can be expressed as

$$
A_{I V}(r, z)=\sum_{k=1}^{\infty} a_{k}^{I V} I_{1}\left(\beta_{k} r\right) \sin \left(\beta_{k} z\right)
$$

where $k$ is a positive odd integer, and $\beta_{k}=k \pi /\left(2 z_{3}\right)$ are the eigenvalues. The integration constant $a_{k}^{I V}$ will be determined in Section 4 from the interface conditions at $r=R_{1}$.

\subsection{General Solution of Laplace's Equation in Region V}

Region $\mathrm{V}$ is delimited by a cylinder of radius $R_{1}$, and by $z=z_{4}$ and $z=z_{5}$. The magnetic vector potential in Region $\mathrm{V}$ is governed by the Laplace equation

$$
\frac{\partial^{2} A_{V}}{\partial r^{2}}+\frac{1}{r} \frac{\partial A_{V}}{\partial r}-\frac{A_{V}}{r^{2}}+\frac{\partial^{2} A_{V}}{\partial z^{2}}=0 \quad \text { for } \quad\left\{\begin{array}{c}
0 \leq r \leq R_{1} \\
z_{4} \leq z \leq z_{5}
\end{array}\right.
$$

The boundary condition at $z=z_{5}$ is

$$
A_{I V}\left(r, z=z_{5}\right)=0
$$

The boundary condition at $z=z_{4}$ is given as for Region IV by

$$
\left.\frac{\partial A_{V}}{\partial z}\right|_{z=z_{4}}=0
$$


Because the magnetic vector potential $A_{V}$ must be finite at $r=0$, and considering the boundary conditions (20) and (21), the general solution of (19) can be expressed as

$$
A_{V}(r, z)=\sum_{k=1}^{\infty} a_{k}^{V} I_{1}\left(\lambda_{k} r\right) \cos \left(\lambda_{k}\left(z-z_{4}\right)\right)
$$

where $k$ is a positive odd integer, and $\lambda_{k}=k \pi /\left(2\left(z_{5}-z_{4}\right)\right)$. The integration constant $a_{k}^{V}$ is to be determinate from the interface conditions at $r=R_{1}$.

The radial and axial flux density distribution in the different regions $(i=I$ to $V$ ) can be deduced from the magnetic vector potential by

$$
B_{i r}=-\frac{\partial A_{i}}{\partial z} \quad \text { and } \quad B_{i z}=\frac{1}{r} \frac{\partial\left(r A_{i}\right)}{\partial r}
$$

\section{INTERFACE CONDITIONS BETWEEN THE REGIONS}

The relations between the integration constants $b_{n}^{I}, a_{n}^{I I}, b_{n}^{I I}, a_{n}^{I I I}$, $b_{n}^{I I I}, a_{k}^{I V}$, and $a_{k}^{V}$ are determined by applying the interface conditions between the different regions. The interface conditions must satisfy the continuity of the radial component of the flux density and the continuity of the axial component of the magnetic field. The first condition could be replaced by the continuity of the magnetic vector potential.

\subsection{Interface Conditions at $r=R_{3}$}

In terms of magnetic vector potential, the interface conditions between Region I and Region II at $r=R_{3}$ lead to:

$$
\begin{aligned}
& A_{I}\left(r=R_{3}, z\right)=A_{I I}\left(r=R_{3}, z\right) \\
& \left.\frac{\partial\left(r A_{I}\right)}{\partial r}\right|_{r=R_{3}}=\left.\frac{\partial\left(r A_{I I}\right)}{\partial r}\right|_{r=R_{3}}
\end{aligned}
$$

From (24), (5), and (10), we obtain two relations between the coefficients of Region I and Region II

$$
\begin{aligned}
& a_{n}^{I I}=\alpha_{n} R_{3} C_{n}\left(L_{0}\left(\alpha_{n} R_{3}\right) K_{1}\left(\alpha_{n} R_{3}\right)+L_{1}\left(\alpha_{n} R_{3}\right) K_{0}\left(\alpha_{n} R_{3}\right)\right) \\
& b_{n}^{I I}=b_{n}^{I}+\alpha_{n} R_{3} C_{n}\left(L_{1}\left(\alpha_{n} R_{3}\right) I_{0}\left(\alpha_{n} R_{3}\right)-L_{0}\left(\alpha_{n} R_{3}\right) I_{1}\left(\alpha_{n} R_{3}\right)\right)
\end{aligned}
$$

where $I_{0}$ and $K_{0}$ are respectively the modified Bessel functions of the first and second kind of order 0 , and $L_{0}$ is the modified Struve function of order 0 . 


\subsection{Interface Conditions at $r=R_{2}$}

The interface conditions between Region II and Region III at $r=R_{2}$ are given by

$$
\begin{aligned}
& A_{I I}\left(r=R_{2}, z\right)=A_{I I I}\left(r=R_{2}, z\right) \\
& \left.\frac{\partial\left(r A_{I I}\right)}{\partial r}\right|_{r=R_{2}}=\left.\frac{\partial\left(r A_{I I I}\right)}{\partial r}\right|_{r=R_{2}}
\end{aligned}
$$

Using (27), (10), and (14), we obtain two relations between the coefficients of Region II and Region III

$$
\begin{aligned}
& a_{n}^{I I I}=a_{n}^{I I}-\alpha_{n} R_{2} C_{n}\left(L_{1}\left(\alpha_{n} R_{2}\right) K_{0}\left(\alpha_{n} R_{2}\right)+L_{0}\left(\alpha_{n} R_{2}\right) K_{1}\left(\alpha_{n} R_{2}\right)\right) \\
& b_{n}^{I I I}=b_{n}^{I I}+\alpha_{n} R_{2} C_{n}\left(L_{0}\left(\alpha_{n} R_{2}\right) I_{1}\left(\alpha_{n} R_{2}\right)-L_{1}\left(\alpha_{n} R_{2}\right) I_{0}\left(\alpha_{n} R_{2}\right)\right)
\end{aligned}
$$

\subsection{Interface Conditions at $r=R_{1}$}

Due to the presence of the iron cylinder (see Fig. 1), the interface condition concerning the axial component of the magnetic field at $r=R_{1}$ is more complex than the ones at $r=R_{2}$ and $r=R_{3}$ and must be divided in three parts. A first part corresponds to the iron cylinder surface $\left(z_{3} \leq z \leq z_{4}\right)$ where the axial component of the magnetic field is null. The other parts corresponds to the continuity of the axial component of the magnetic field between Region III and Region IV and between Region III and Region V. Therefore, we can write

$$
\left.\frac{\partial\left(r A_{I I I}\right)}{\partial r}\right|_{R_{1}}= \begin{cases}\left.\frac{\partial\left(r A_{I V}\right)}{\partial r}\right|_{R_{1}} & \forall z \in\left[0, z_{3}\right] \\ 0 & \forall z \in\left[z_{3}, z_{4}\right] \\ \left.\frac{\partial\left(r A_{V}\right)}{\partial r}\right|_{R_{1}} & \forall z \in\left[z_{4}, z_{5}\right]\end{cases}
$$

The continuity of the radial component of the flux density yields to

$$
\begin{array}{rll}
A_{I V}\left(r=R_{1}, z\right) & =A_{I I I}\left(r=R_{1}, z\right) & \forall z \in\left[0, z_{3}\right] \\
A_{V}\left(r=R_{1}, z\right) & =A_{I I I}\left(r=R_{1}, z\right) & \forall z \in\left[z_{4}, z_{5}\right]
\end{array}
$$

From (30), (31), (32) and using the Fourier series method, we obtain three equations between the coefficients of Regions III, IV, and $\mathrm{V}$

$$
\begin{aligned}
& a_{n}^{I I I}-b_{n}^{I I I} \frac{K_{0}\left(\alpha_{n} R_{1}\right)}{I_{0}\left(\alpha_{n} R_{1}\right)} \\
= & \sum_{k=1}^{\infty}\left(a_{k}^{I V} \frac{k}{n z_{3}} \frac{I_{0}\left(\beta_{k} R_{1}\right)}{I_{0}\left(\alpha_{n} R_{1}\right)} f(n, k)+a_{k}^{V} \frac{k}{n\left(z_{5}-z_{4}\right)} \frac{I_{0}\left(\lambda_{k} R_{1}\right)}{I_{0}\left(\alpha_{n} R_{1}\right)} g(n, k)\right)
\end{aligned}
$$




$$
\begin{aligned}
a_{k}^{I V} & =\frac{2}{z_{3}} \sum_{n=1}^{\infty}\left(a_{n}^{I I I} \frac{I_{1}\left(\alpha_{n} R_{1}\right)}{I_{1}\left(\beta_{k} R_{1}\right)}+b_{n}^{I I I} \frac{K_{1}\left(\alpha_{n} R_{1}\right)}{I_{1}\left(\beta_{k} R_{1}\right)}\right) \times f(n, k) \\
a_{k}^{V} & =\frac{2}{z_{5}-z_{4}} \sum_{n=1}^{\infty}\left(a_{n}^{I I I} \frac{I_{1}\left(\alpha_{n} R_{1}\right)}{I_{1}\left(\lambda_{k} R_{1}\right)}+b_{n}^{I I I} \frac{K_{1}\left(\alpha_{n} R_{1}\right)}{I_{1}\left(\lambda_{k} R_{1}\right)}\right) \times g(n, k)
\end{aligned}
$$

where

$$
\begin{aligned}
& f(n, k)=\left\{\begin{array}{lll}
-\frac{\alpha_{n} \sin (k \pi / 2) \cos \left(\alpha_{n} z_{3}\right)}{\left(\alpha_{n}^{2}-\beta_{k}^{2}\right)} & \text { for } & \alpha_{n} \neq \beta_{k} \\
0.5 \times z_{3} & \text { for } & \alpha_{n}=\beta_{k}
\end{array}\right. \\
& g(n, k)=\left\{\begin{array}{lll}
-\frac{\alpha_{n} \cos \left(\alpha_{n} z_{4}\right)}{\left(\alpha_{n}^{2}-\lambda_{k}^{2}\right)} & \text { for } \quad \alpha_{n} \neq \lambda_{k} \\
0.5 \times\left(z_{5}-z_{4}\right) \sin \left(\alpha_{n} z_{4}\right) & \text { for } & \alpha_{n}=\lambda_{k}
\end{array}\right.
\end{aligned}
$$

Because $a_{n}^{I I}$ and $a_{n}^{I I I}$ are directly linked to the source term (25) and (28), we have only to solve a system of five linear equations with five unknowns. By rewriting the above equations in matrix and vectors format, a numerical solution can be found by using mathematical software (Matlab, Mathematica, etc.). It should be noted here that a numerical matrix inversion is required for the calculation of the unknown coefficients but using symbolic packages, this matrix needs to be inverted only once even in parametric studies.

\section{SELF-INDUCTANCE AND ELECTROMAGNETIC FORCE EXPRESSION}

\subsection{Electromagnetic Force Expression}

The electromagnetic force acting on the iron core is obtained using the Maxwell stress tensor method. A line of radius $R_{e}$ and length $\left[0, z_{5}\right]$ in Region III is taken as the integration path (it is also possible to choice an integration path directly around the iron core but we obtain a more complex analytical expression). The electromagnetic force in the axial direction can be expressed as follows

$$
F_{z}=\frac{2 \pi R_{e}}{\mu_{0}} \int_{0}^{z_{5}} B_{I I I r}\left(R_{e}, z\right) B_{I I I z}\left(R_{e}, z\right) d z
$$

where $B_{I I I r}$ and $B_{I I I z}$ are respectively the radial and the axial components of the flux density in Region III. Their expressions can be obtained from (14) and (23)

$$
B_{I I I r}(r, z)=\sum_{n=1}^{\infty}-\alpha_{n}\left\{a_{n}^{I I I} I_{1}\left(\alpha_{n} r\right)+b_{n}^{I I I} K_{1}\left(\alpha_{n} r\right)\right\} \cos \left(\alpha_{n} z\right)
$$




$$
B_{I I I z}(r, z)=\sum_{n=1}^{\infty} \alpha_{n}\left\{a_{n}^{I I I} I_{0}\left(\alpha_{n} r\right)-b_{n}^{I I I} K_{0}\left(\alpha_{n} r\right)\right\} \sin \left(\alpha_{n} z\right)
$$

Substituting (39) and (40) into (38), we obtain the following analytical expression for the electromagnetic force

$$
F_{z}=\frac{4 z_{5} R_{e}}{\mu_{0}} \sum_{n=1}^{\infty} \sum_{m=1}^{\infty} \frac{m}{m^{2}-n^{2}} X_{n} Y_{m} \quad \text { with } \quad m \neq n
$$

and

$$
\begin{aligned}
& X_{n}=-\alpha_{n}\left\{a_{n}^{I I I} I_{1}\left(\alpha_{n} R_{e}\right)+b_{n}^{I I I} K_{1}\left(\alpha_{n} R_{e}\right)\right\} \\
& Y_{m}=\alpha_{m}\left\{a_{m}^{I I I} I_{0}\left(\alpha_{m} R_{e}\right)-b_{m}^{I I I} K_{0}\left(\alpha_{m} R_{e}\right)\right\}
\end{aligned}
$$

where $m$ and $n$ are positive integers.

\subsection{Self-inductance Expression}

The self-inductance $L_{11}$ of the iron-core solenoid is related to the total stored magnetic energy as

$$
\frac{1}{2} L_{11} I^{2}=\frac{1}{2} \int_{V} A \cdot J d v
$$

The integral in (43) is restricted to the volume of the conductor, since the current density elsewhere is zero. We have supposed that the current density is uniformly distributed over the whole cross section of the winding, this leads to

$$
I=\frac{J\left(R_{3}-R_{2}\right) L}{N}
$$

where $N$ and $I$ are the number of turns in the winding and the electrical current in the wire, respectively. $L$ is the axial length of the coil.

The magnetic vector potential in the winding is given by (10). Substituting (10) and (44) into (43) and integrating first in respect to the $z$ variable, we obtained

$$
\begin{aligned}
L_{11}= & \frac{2 \pi N^{2}}{\left(R_{3}-R_{2}\right)^{2} L^{2} J} \sum_{n=1}^{\infty}\left\{\frac{\cos \left(\alpha_{n} z_{1}\right)-\cos \left(\alpha_{n} z_{2}\right)}{\alpha_{n}}\right. \\
& \left.\times \int_{R_{2}}^{R_{3}}\left(a_{n}^{I I} I_{1}\left(\alpha_{n} r\right)+b_{n}^{I I} K_{1}\left(\alpha_{n} r\right)-C_{n} L_{1}\left(\alpha_{n} r\right)\right) r d r\right\}
\end{aligned}
$$

The radial integration in (45) leads to integral of the form:

$$
\int r I_{1}\left(\alpha_{n} r\right) d r=\frac{\pi r}{2 \alpha_{n}}\left(I_{1}\left(\alpha_{n} r\right) L_{0}\left(\alpha_{n} r\right)-I_{0}\left(\alpha_{n} r\right) L_{1}\left(\alpha_{n} r\right)\right)=\frac{\pi r}{2 \alpha_{n}} \mathrm{U}(r)
$$




$$
\begin{aligned}
& \int r K_{1}\left(\alpha_{n} r\right) d r=\frac{\pi r}{2 \alpha_{n}}\left(K_{1}\left(\alpha_{n} r\right) L_{0}\left(\alpha_{n} r\right)+K_{0}\left(\alpha_{n} r\right) L_{1}\left(\alpha_{n} r\right)\right)=\frac{\pi r}{2 \alpha_{n}} \mathrm{~V}(r)(47) \\
& \int r L_{1}\left(\alpha_{n} r\right) d r=\frac{\alpha_{n}^{2} r^{4}}{6 \pi} F\left(1,2 ; \frac{3}{2}, \frac{5}{2}, 3 ; \frac{\alpha_{n}^{2} r^{2}}{4}\right)=\frac{\alpha_{n}^{2} r^{4}}{6 \pi} \mathrm{W}(r)
\end{aligned}
$$

where $F$ in (48) is the hypergeometric function [28]. The functions $U(r), V(r)$ and $W(r)$ in (46) to (48) have been introduced here to simplify the mathematical expressions. Substituting (46), (47) and (48) into (45), the semi-analytical expression of the self-inductance is given by:

$$
\begin{aligned}
& L_{11}=\frac{2 \pi N^{2}}{\left(R_{3}-R_{2}\right)^{2} L^{2} J} \\
& \sum_{n=1}^{\infty}\left\{\frac{\cos \left(\alpha_{n} z_{1}\right)-\cos \left(\alpha_{n} z_{2}\right)}{2} \times\left(\begin{array}{l}
a_{n}^{I I} \frac{\pi}{\alpha_{n}^{2}}\left(R_{3} U\left(R_{3}\right)-R_{2} U\left(R_{2}\right)\right) \\
+b_{n}^{I I} \frac{\pi}{\alpha_{n}^{2}}\left(R_{3} V\left(R_{3}\right)-R_{2} V\left(R_{2}\right)\right) \\
-C_{n} \frac{\alpha_{n}}{3 \pi}\left(R_{3}^{4} W\left(R_{3}\right)-R_{2}^{4} W\left(R_{2}\right)\right)
\end{array}\right)\right\}
\end{aligned}
$$

An analytical expression for the self-inductance without the iron core can be obtained by imposing the coefficients $b_{n}^{I I I}, a_{k}^{I V}$, and $a_{k}^{V}$ to be null (Regions IV and V disappears without the ferromagnetic rod), that gives from (25) and (29)

$$
a_{n}^{I I}=\alpha_{n} R_{3} C_{n} V\left(R_{3}\right) \quad b_{n}^{I I}=-\alpha_{n} R_{2} C_{n} U\left(R_{2}\right)
$$

By substituting (11) and (50) into (49), we obtain an analytical expression for the self-inductance without the iron-core in term of an infinite series as:

$$
\begin{aligned}
& L_{11}^{\prime}= \frac{4 \mu_{0} N^{2} R_{3}^{2} z_{5}^{3}}{\pi\left(R_{3}-R_{2}\right)^{2} L^{2}} \sum_{n=1,3,5 \ldots}^{\infty} \frac{1}{n^{4}} \sin ^{2}\left(\frac{n \pi L}{2 z_{5}}\right) \\
& \times\left(\begin{array}{l}
U\left(R_{3}\right) V\left(R_{3}\right)-2 \frac{R_{2}}{R_{3}} U\left(R_{2}\right) V\left(R_{3}\right) \\
+\left(\frac{R_{2}}{R_{3}}\right)^{2} U\left(R_{2}\right) V\left(R_{2}\right) \\
-\frac{\left(n R_{3}\right)^{2}}{3 z_{5}^{2}}\left(W\left(R_{3}\right)-\left(\frac{R_{2}}{R_{3}}\right)^{4} W\left(R_{2}\right)\right)
\end{array}\right)
\end{aligned}
$$

\section{ANALYTICAL RESULTS AND COMPARISON WITH FINITE ELEMENT SIMULATIONS}

The geometrical parameters are given in Table 1 . The outer boundaries in the axial direction have been placed at $z=0 \mathrm{~cm}$ and $z_{5}=200 \mathrm{~cm}$. These boundaries are sufficiently far away from the coil and the iron core so that they do not affect the results (the length of the domain is 


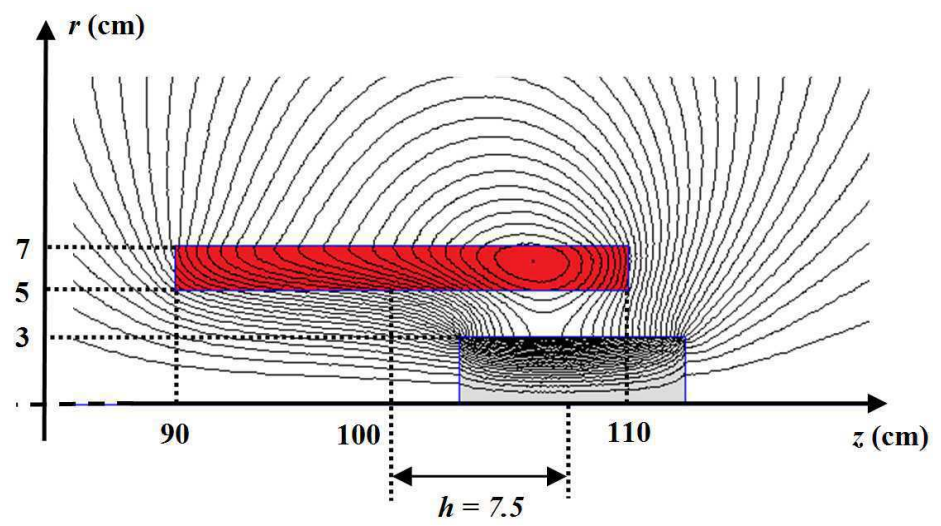

Figure 3. Equipotential lines around the coil for $h=7.5 \mathrm{~cm}$ (maximum force).

ten times bigger than the length of the coil). Analytical solutions in Regions I to $\mathrm{V}$ have been computed with a finite number of harmonic terms $M$ and $K$ as indicated in Table 1 .

In order to validate the proposed model, the analytical results have been compared with those obtained by using a finite element (FE) software FEMM [30]. For the FE solutions, a relative permeability of $\mu_{r}=10000$ has been used for the iron core. The axial length of the whole domain in FE simulations is the same as the one of the analytical model. Homogeneous Dirichlet boundary conditions have been imposed at $z=0$ and $z=z_{5}$ for FE simulations like for the analytical model. The mesh in the different regions has been refined until convergent results are obtained.

Figure 3 shows the equipotential lines around the coil when the center of the iron core is placed at a distance $h=7.5 \mathrm{~cm}$ from the center of the coil. This position corresponds to the maximum force acting on the iron core as it can be observed in Fig. 5 .

The radial and axial components of the magnetic flux density distribution along the $z$-axis in Region III are shown in Fig. 4. The flux density distribution without the iron core is also plotted in this figure (when the iron core is not present, the solution is symmetric about the center of the coil). The results without the iron core are obtained with the analytical model by imposing the coefficients $b_{n}^{I I I}, a_{k}^{I V}$, and $a_{k}^{V}$ to be null.

From Fig. 4, the effects of the iron core on the magnetic field distribution are very clear. One can see the distortion of the flux density waveforms at the vicinity of the iron cylinder. An excellent 
agreement with the results deduced from FEM is obtained.

Figure 5 compares the electromagnetic axial force acting on the iron core obtained with the semi-analytical formula (41) and with FE simulations. For the computation, the center of the iron core is displaced axially by a distance $h$ relative to the center of the coil. As expected, the force is null when the iron core is centered inside the coil and it presents a symmetry around $h=0$. The maximum force is reached for a displacement $h=7.5 \mathrm{~cm}$ and its value is equal to $76.8 \mathrm{~N}$ with the analytical model and $77.9 \mathrm{~N}$ with FEM. The error is less than $1.5 \%$ if we considered 50 harmonic terms. It appears that results from the proposed analytical method and $\mathrm{FE}$ simulations are very close to each other. To compute the peak value of the force, the computation time is $1.21 \mathrm{~s}$ with the analytical model (50 harmonic terms, Processor Intel Core2 Duo P8700, 2.53 GHz, Matlab Software) whereas the finite element simulation takes $5.83 \mathrm{~s}$ for a mesh of 97423 elements [30]. The

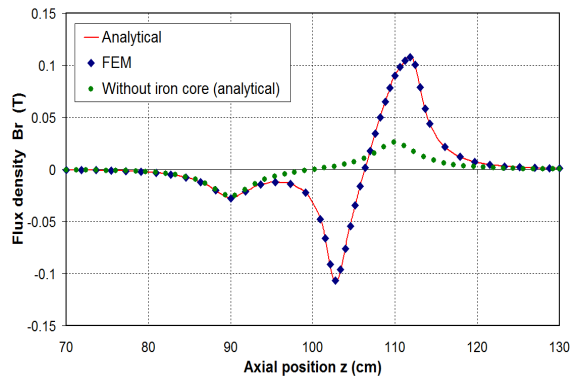

(a)

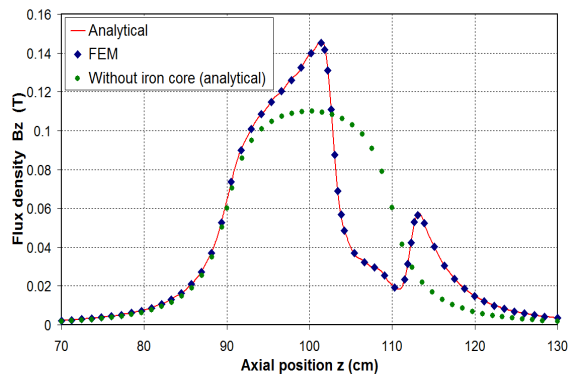

(b)

Figure 4. Radial (a) and axial (b) components of the flux density in Region III for $r=4 \mathrm{~cm}$ and $h=7.5 \mathrm{~cm}$.

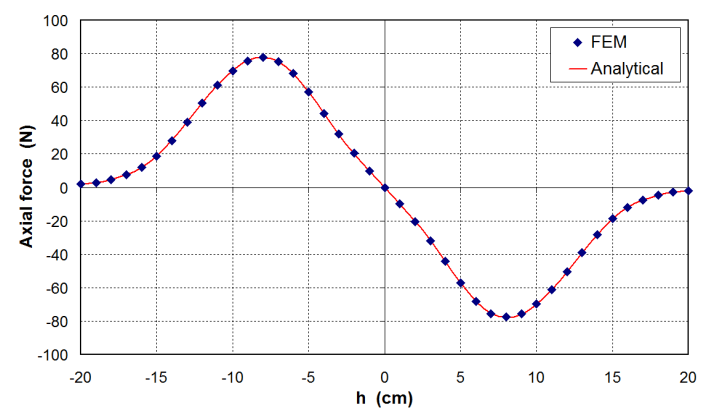

Figure 5. Electromagnetic force versus iron-core position $h$. 
Table 1. Geometrical parameters.

\begin{tabular}{|c|c|c|}
\hline Symbol & Quantity & value \\
\hline$R_{1}$ & Radius of the iron core & $3 \mathrm{~cm}$ \\
\hline$R_{2}$ & Inner radius of the coil & $5 \mathrm{~cm}$ \\
\hline$R_{3}$ & Outer radius of the coil & $7 \mathrm{~cm}$ \\
\hline$z_{1}$ & Axial position of the coil (left side) & $90 \mathrm{~cm}$ \\
\hline$z_{2}$ & Axial position of the coil (right side) & $110 \mathrm{~cm}$ \\
\hline$L$ & Axial length of the coil $\left(L=z_{2}-z_{1}\right)$ & $20 \mathrm{~cm}$ \\
\hline$N$ & Number of turns in the winding & 1000 \\
\hline$h$ & $\begin{array}{l}\text { Axial position of the center of } \\
\text { the iron core from the center of the coil }\end{array}$ & variable \\
\hline$z_{3}$ & Axial position of the iron core (left side) & $95+h \mathrm{~cm}$ \\
\hline$z_{4}$ & Axial position of the iron core (right side) & $105+h \mathrm{~cm}$ \\
\hline$l$ & Axial length of the iron core $\left(l=z_{4}-z_{3}\right)$ & $10 \mathrm{~cm}$ \\
\hline$z_{5}$ & Outer boundary of the domain & $200 \mathrm{~cm}$ \\
\hline$J$ & Current density in the coil & $5 \mathrm{~A} / \mathrm{mm}^{2}$ \\
\hline$M$ & $\begin{array}{l}\text { Number of harmonic terms used for magnetic } \\
\text { field calculation in Regions I, II and III }\end{array}$ & 50 \\
\hline$K$ & $\begin{array}{l}\text { Number of harmonic terms used for magnetic } \\
\text { field calculation in Regions IV and V }\end{array}$ & 50 \\
\hline
\end{tabular}

analytical computation being much faster, the presented model can advantageously be used in a preliminary design stage.

Figure 6 gives the self-inductance variation versus iron rod position obtained with the semi-analytical formula (49) and with FE simulations. As for the magnetic force, the iron core is displaced axially by a distance $h$ relative to the center of the coil. As expected, the selfinductance is maximal for $h=0 \mathrm{~cm}$ and presents a symmetry around $h=0 \mathrm{~cm}$. The maximal value of the self-inductance is $L_{11}=85.8 \mathrm{mH}$ with the analytical model ( 50 harmonic terms) and $L_{11}=87 \mathrm{mH}$ with finite element simulations (97 423 elements). The error is less than $1.4 \%$. The value of the self-inductance without the iron core (51) is $L_{11}^{\prime}=48.8 \mathrm{mH}$ by using the analytical model (50 harmonics) and $L_{11}^{\prime}=49 \mathrm{mH}$ with finite element simulations. Without the iron cylinder (51), the error on the self-inductance computation is less than $0.4 \%$ and the computational time is much faster $(0.11 \mathrm{~s}$ for 50 harmonic terms). The analytical expressions for the self-inductance with or without iron core are well verified by comparison with finite 


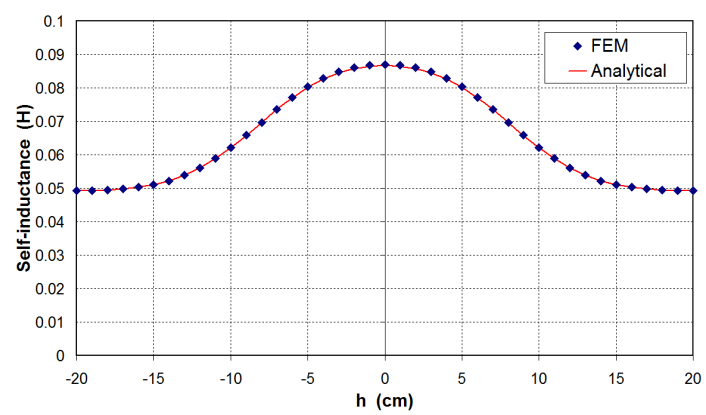

Figure 6. Self-inductance versus iron-core position $h$.

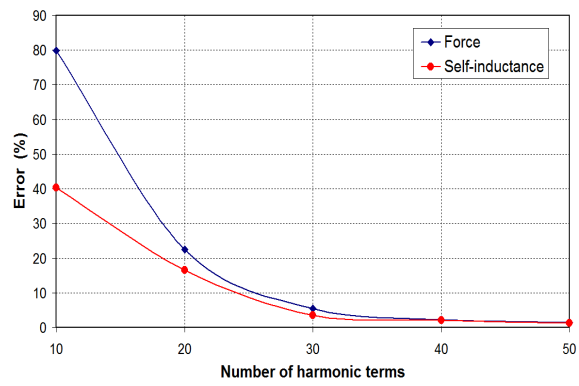

(a)

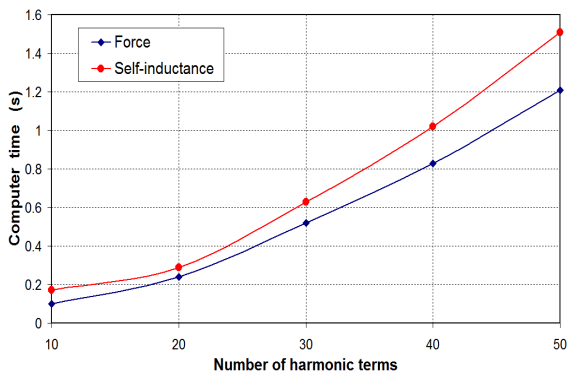

(b)

Figure 7. Performances of the analytical model versus the number of harmonic terms; (a) Error; (b) Computer time.

element simulations.

The error (axial force and self-inductance values) and computer time variations with respect to the number of harmonic terms considered in evaluating the analytical solutions are given in Table 2 and are shown in Fig. 7(a) and Fig. 7(b). The same number of harmonic terms is considered for the five regions $(M=K)$. For the definition of the error, we assumed that results obtained with finite element simulations are correct $\left(F=77.9 \mathrm{~N}\right.$ and $\left.L_{11}=87 \mathrm{mH}\right)$. Only the peak values of the force $(h=7.5 \mathrm{~cm})$ and self-inductance $(h=0 \mathrm{~cm})$ are considered here. As shown in Fig. 7, the error increases and the computer time decreases when the number of harmonic terms decreases. We can observe from Fig. 7(a) that the error is smaller for the self-inductance than for the force. From Table 2, we can say that a number of 40 harmonic terms in the analytical model seems to be a good compromise in terms of precision and computer time for the studied example. 
Table 2. Error and computer time variations with respect to the number of harmonic terms.

\begin{tabular}{|c|c|c|c|c|c|c|}
\hline \multicolumn{2}{|c|}{ Number of harmonic terms } & $\mathbf{5 0}$ & $\mathbf{4 0}$ & $\mathbf{3 0}$ & $\mathbf{2 0}$ & $\mathbf{1 0}$ \\
\hline \multirow{4}{*}{ Force } & Value $(\mathrm{N})$ & 76.8 & 76.1 & 73.6 & 60.3 & 15.7 \\
\cline { 2 - 7 } & Error: $\varepsilon(\%)$ & 1.4 & 2.3 & 5.5 & 22.5 & 79.8 \\
\cline { 2 - 7 } & Computer time: $T(\mathrm{~s})$ & 1.21 & 0.83 & 0.52 & 0.24 & 0.1 \\
\cline { 2 - 7 } & Figure of merit: $\varepsilon \cdot T$ & 1.69 & 1.9 & 2.86 & 5.4 & 7.98 \\
\hline \multirow{4}{*}{$\begin{array}{c}\text { Self- } \\
\text { inductance }\end{array}$} & Value $(\mathrm{mH})$ & 85.8 & 85.2 & 83.9 & 72.6 & 51.9 \\
\cline { 2 - 7 } & Error: $\varepsilon(\%)$ & 1.3 & 2.1 & 3.6 & 16.5 & 40.3 \\
\cline { 2 - 7 } & Computer time: $T(\mathrm{~s})$ & 1.51 & 1.02 & 0.63 & 0.29 & 0.17 \\
\cline { 2 - 7 } & Figure of merit: $\varepsilon \cdot T$ & 1.96 & 2.14 & 2.26 & 4.78 & 6.85 \\
\hline
\end{tabular}

We can define the figure of merit of the proposed analytical model as the product of the error by the computer time. Figure of merit (for the force and self-inductance evaluation) is investigated with varying number of harmonics. The results are given in Table 2. More the figure of merit is low, better is the performance of the analytical model. From Table 2, we can observe that the figure of merit is better for large value of harmonic terms. The figure of merit for the self-inductance computation is practically constant (around a value of 2 ) for a number of harmonic terms upper than 30 .

\section{CONCLUSION}

In this paper, we have developed new semi-analytical expressions to compute the self-inductance and electromagnetic force for iron-core solenoid of finite length. The analytical model is based on boundary value problems with Fourier analysis. The Laplace and the Poisson equations have been solved in the different regions by the separation of variable methods. The solutions in each region are given in terms of infinite series.

The only approximation in the modeling of this problem is in assuming infinite permeability for the iron cylinder. The accuracy of the analytical expressions for the self-inductance and the electromagnetic force has been verified through comparisons with numerical results from FE simulations. The proposed model can be extended easily to multi-layers circular coils systems with or without iron-core. 


\section{REFERENCES}

1. Garrett, M. W., "Calculations of fields, forces and mutual inductances of current systems by elliptic integral," J. Appl. Phys., Vol. 34, 2567, 1963.

2. Durand, E., Magnétostatique, Masson et Cie, Paris, 1968.

3. Urankar, L., "Vector potential and magnetic field of currentcarrying finite arc segment in analytical form, part III: Exact computation for rectangular cross-section," IEEE Trans. Magn., Vol. 18, No. 6, 1860-1867, Nov. 1982.

4. Yu, D. and K. S. Han, "Self-inductance of aire-core circular coils with rectangular cross-section," IEEE Trans. Magn., Vol. 43, No. 6, 3916-3921, Nov. 1987.

5. Rezzoug, A., J. P. Caron, and F. M. Sargos, "Analytical calculation of flux induction and forces of thick coils with finite length," IEEE Trans. Magn., Vol. 28, No. 5, 2250-2252, Sep. 1992.

6. Azzerboni, B., E. Cardelli, M. Raugi, A. Tellini, and G. Tina, "Magnetic field evaluation for thick annular conductors," IEEE Trans. Magn., Vol. 29, No. 3, 2090-2094, May 1993.

7. Conway, J. T., "Exact solutions for the magnetic fields of solenoids and current distributions," IEEE Trans. Magn., Vol. 37, No. 4, 2977-2988, Jul. 2001.

8. Babic, S. and C. Akiel, "Improvement of the analytical calculation of the magnetic field produced by permanent magnet rings," Progress In Electromagnetic Research C, Vol. 5, 71-82, 2008.

9. Conway, J. T., "Trigonometric integrals for the magnetic field of the coil of rectangular cross section," IEEE Trans. Magn., Vol. 42, No. 5, 1538-1548, May 2006.

10. Babic, S. and C. Akyel, "Magnetic force calculation between thin coaxial circular coils in air," IEEE Trans. Magn., Vol. 44, No. 4, 445-452, Apr. 2008.

11. Conway, J. T., "Inductance calculations for circular coils of rectangular cross section and parallel axes using Bessel and Struve functions," IEEE Trans. Magn., Vol. 46, No. 1, 75-81, Jan. 2010.

12. Ravaud, R., G. Lemarquand, V. Lemarquand, and C. Depollier, "The three exact components of the magnetic field created by a radially magnetized tile permanent magnet," Progress In Electromagnetics Research, Vol. 88, 307-319, 2008.

13. Ravaud, R., G. Lemarquand, V. Lemarquand, S. Babic, and C. Akyel, "Mutual inductance and force exerted between thick coils," Progress In Electromagnetics Research, Vol. 102, 367-380, 
2010.

14. Babic, S., F. Sirois, C. Akyel, G. Lemarquand, V. Lemarquand, and R. Ravaud, "New formulas for mutual inductance and axial magnetic force between a thin wall solenoid and a thick circular coil of rectangular cross-section," IEEE Trans. Magn., Vol. 47, No. 8, 2034-2044, Aug. 2011.

15. Zhang, D. and C. S. Koh, "An efficient semi-analytic computation method of magnetic field for a circular coil with rectangular cross section," IEEE Trans. Magn., Vol. 48, No. 1, 62-68, Jan. 2012.

16. Gysen, B. L. J., K. J. Meessen, J. J. H. Paulides, and E. A. Lomonova, "General formulation of the electromagnetic field distribution in machines and devices using Fourier analysis," IEEE Trans. Magn., Vol. 46, No. 1, 39-52, Jan. 2010.

17. Lubin, T., S. Mezani, and A. Rezzoug, "Exact analytical method for magnetic field computation in the air-gap of cylindrical electrical machines considering slotting effects," IEEE Trans. Magn., Vol. 46, No. 4, 1092-1099, Apr. 2010.

18. Rabins, L., "Transformer reactances calculation with digital computer," AIEE Trans., Vol. 75, Pt. I, 261-267, Jul. 1956.

19. Martinelli, G. and A. Morini, "A potential vector field solution in superconducting magnets, part I: Method of calculation," IEEE Trans. Magn., Vol. 19, No. 4, 1537-1545, Jul. 1983.

20. Caldwell, J. and A. Zisserman, "A Fourier series approach to magnetostatic field calculations involving magnetic materials," $J$. Appl. Phys., Vol. 54, No. 9, 4734-4738, Sep. 1983.

21. Caldwell, J. and A. Zisserman, "Magnetostatic field calculations involving iron using eigenfunction expansion," IEEE Trans. Magn., Vol. 19, No. 6, 2725-2729, Nov. 1983.

22. Uzal, E., I. Ozkol, and M. O. Kaya, "Impedance of a coil surrounding an infinite cylinder with an arbitrary radial conductivity profile," IEEE Trans. Magn., Vol. 34, No. 1, 213217, Nov. 1987.

23. Wilcox, D. J., M. Conlon, and W. G. Hurley, "Calculation of self and mutual impedances for coils on ferromagnetic cores," IEE Proceedings, Vol. 135, Pt. A, No. 7, 470-476, Jan. 1998.

24. Dodd, C. V. and W. E. Deeds, "Analytical solutions to eddycurrent probe-coil problems," Journal of Applied Physics, Vol. 39, 2829-2838, May 1968.

25. Dodd, C. V., C. C. Cheng, and W. E. Deeds, "Induction coils coaxial with an arbitrary number of cylindrical conductors," Journal of Applied Physics, Vol. 45, 638-647, Feb. 1974. 
26. Bowler, J. R. and T. P. Theodoulidis, "Eddy currents induced in a conducting rod of finite length by a coaxial encircling coil," Journal of Physics D: Applied Physics, Vol. 38, 2861-2868, 2005.

27. Sun, H., J. R. Bowler, and T. P. Theodoulidis, "Eddy currents induced in a finite length layered rod by a coaxial coil," IEEE Trans. Magn., Vol. 41, No. 9, 2455-2461, Sep. 2005.

28. Abramowitz, M. and I. A. Stegun, Handbook of Mathematical Functions, Dover Publications, Inc., New York, 1972.

29. Farlow, S. J., Partial Differential Equations for Scientists and Engineers, 414, Dover Publications, New York, 1993.

30. Meeker, D. C., Finite Element Method Magnetics, Version 4.2, Apr. 1, 2009, http://www.femm.info. 